\title{
Stochastic model for predicting the bending strength of glued- laminated timber based on the knot area ratio and localized MOE in lamina
}

\author{
Sung-Jun Pang ${ }^{1} \cdot$ Jung-Kwon $\mathrm{Oh}^{2} \cdot$ Jung-Pyo Hong $^{3} \cdot$ Sang-Joon Lee ${ }^{4} \cdot J_{\text {Jun-Jae Lee }}{ }^{2}$
}

Received: 17 May 2017 / Accepted: 16 November 2017 / Published online: 5 January 2018

(c) The Japan Wood Research Society 2018

\begin{abstract}
The aim of this study was to develop a stochastic model for predicting the bending strength distribution of glued-laminated timber (GLT). The developed model required the localized modulus of elasticity (MOE) and tensile strengths of laminae as input properties. The tensile strength was estimated using a regression model based on the localized MOEs and knot area ratios (KAR) which were experimentally measured for lamina grades samples. The localized MOE was obtained using a machine stress-rated grader, and the localized KAR was determined using an image-processing system. The bending strength distributions in four types of GLTs were simulated using the developed GLT beam model; these four types included: (1) GLT beams without finger joints; (2) GLT beams with finger joints; (3) GLT beams with different lamina sizes; and (4) GLT beams with different combinations of lamina grades. The simulated bending strength distributions were compared with actual test data of 2.4 and 4.8 m-long GLTs. The Kolmogorov-Smirnov goodness-of-fit tests showed that all of the simulated bending strength distributions agreed well with the test data. Especially, good agreement was shown in the fifth percentile point estimate of bending strength with the difference of approximately $1 \%$.
\end{abstract}

Keywords Glued-laminated timber $\cdot$ Stochastic model $\cdot$ Bending strength distribution $\cdot$ Knot area ratio

\section{Introduction}

Glued-laminated timber (GLT) is widely used as a building material, and its characteristic value used in structural design is determined by identifying the fifth percentile of the GLT

This article was presented in 2016 World Conference on Timber Engineering.

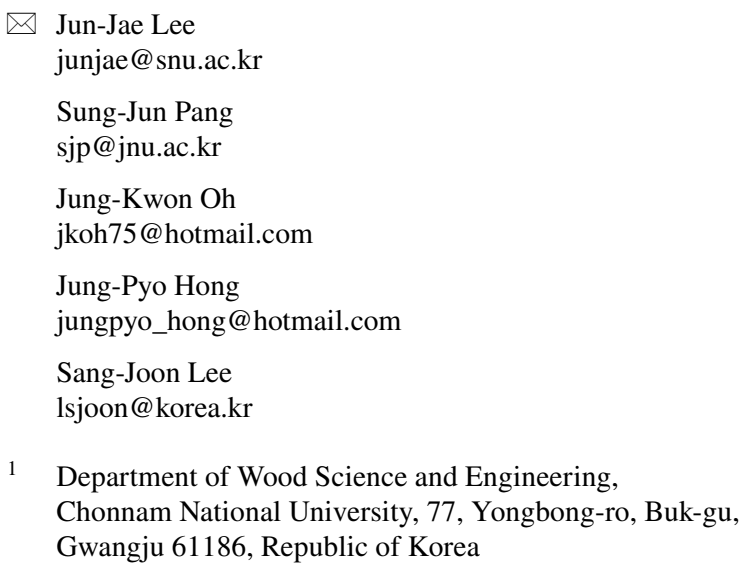

1 Department of Wood Science and Engineering, Chonnam National University, 77, Yongbong-ro, Buk-gu, Gwangju 61186, Republic of Korea

strength distribution. In general, to obtain the distribution, enormous experimental tests are required for GLTs with various lamina combinations and grades. Thus, the experimental tests are not a practical approach to derive the whole strength distributions for various GLT grades. To estimate the population statistics for GLT beams, computer simulation models [1-5] have been efficiently used. The basic

2 Department of Forest Sciences, Research Institute for Agriculture and Life Sciences, Seoul National University, 6218-200dong 599 Gwanak-ro, Gwanak-gu, Seoul 08826, Republic of Korea

3 Korea Forestry Promotion Institute, 475 Gonghang-daero, Gangseo-gu, Seoul 07570, Republic of Korea

4 Department of Forest Products, National Institute of Forest Science, 57 Heogiro, Dongdaemun-gu, Seoul 02455, Republic of Korea 
concept of the presented models is similar and based on beam theory.

The lamina is usually graded by a weak section, especially the size of knot, or the weakest modulus of elasticity (MOE) of the lamina. In the manufacturing process, if a lamina does not meet the visual quality requirement for a certain grade but the lamina satisfies the MOE criterion, the manufacturer can remove the knot then make finger joint. Since the manufacturer can perform the visual quality control for the lamina products, the manufacturer should know what the level of visual quality would be appropriate to manufacturing GLTs. To reflect this manufacturing process in a GLT beam model, the knot size variation and knot position in the longitudinal direction of lamina should be analyzed. It is difficult to measure and record the knot information by hand. However, nowadays, the image-processing system has enabled the process to be done automatically.

In this study, a new GLT beam model was developed based on the actual knot size and location in lamina recorded by an image-processing system. In addition, at the detected position of knot in a lamina, the corresponding MOE recorded by machine stress-rated (MSR) grader was used to estimate the tensile strength of the lamina. For the model validation, the mechanical bending tests of GLTs were conducted.

\section{Materials and methods}

\section{Basic concept}

In this paper, the model was developed to estimate the bending strength distributions of GLTs which were manufactured under different conditions; the number of layup, the grade combination, and the probabilistic properties of the corresponding lamina grade. In this development, the following were considered.

1. Size/volume effect: GLT is a laminated product and it can be made in a large volume. For a large GLT, it is impractical to measure fifth percentile of bending strength by experimental approach in viewpoint of efficiency. In this study, the model development was intended to be capable of capturing the size/volume effect of GLT. To do this, the concept of the weakest link theory, which addresses this effect statistically, was incorporated into the model. Each lamina model was segmented along the length into $1 \mathrm{~mm}$-long elements which were assumed as the links in the theory. For every elements (or links), the lamina material properties were statistically generated in the GLT model. Then, the bending strength of GLT model was determined by computing the stress profile of the elements.
2. Knot size: the mechanical properties of lamina would be different along the length. This variation would make effects on the bending strength of the GLT in the end. Therefore, the MOE and strength properties of each element along the length are essential measurements for predicting bending strength of GLT. However, the measurement of the fully-lengthwise properties is not practical in reality. Only a limited length of lamina can be used for the measurement because of testing apparatus. For an example, in the tension test, some portions of specimen in the length were damaged by tension grips. Therefore, in this study, knot size, which was the most influential defect on the strength, was selected and measured as a strength estimator. Based on the measurements of the localized MOE and knot size, the strength was computed.

3. Input properties: the performance of scanning system has been quickly improved. For knot size information, charge-coupled device (CCD)-camera scanning technique can be used and also MSR grader would be a good way to measure localized MOE. In this study, CCD-camera scanning system and MSR-grading system were used. These machine systems can provide an efficient and consistent way to make the database of input properties for each lamina grade.

In this paper, the model was developed under the assumptions below.

1. Failure mode: there are many reports that the GLT is failed by tension at the location of knot or finger joint under bending stress [6]. To simplify the model, only the tension failure was assumed in this study. In reality, some compression failure could occur in compression side of beam. However, the influence of compression side was not considered in the model development.

2. Elastic behavior: under the first assumption, the behavior under tension was assumed as elastic.

3. Finger joint: a manufacturer might make a finger joint when a knot larger than visual quality (VQ) restraint was found, or some serious defects/drying problem should be removed. In this study, knot was focused. Hence, if there is a larger knot than predefined knot restraint, the portion of the knot in a lamina was removed and finger-jointed.

\section{Lamina database}

For a lamina model, the MOE and tensile strength profile were generated by a material model which was developed with the measured knot area ratio (KAR), the ratio of knot area to the cross-sectional area, and MOE database. Because the MOE and tensile strength must be correlated, 
the database was developed for the two-measured properties to be on a same position along the length of lamina.

\section{Assigning the input properties from the lamina database}

In GLT manufacturing, the manufacturer calculates the required number of lamina. From the calculation, the manufacturer lists up the number of lamina for each grade. The over-sized knots than the restraint could be cutoff. Then, the remnant laminae were to be finger-jointed. When the finger-jointed lamina was longer than the finished length of GLT, the finger-jointed lamina would be cut to the length of GLT. Likewise, the computer model was developed to follow the same manufacturing process. The first input properties of lamina grade required were randomly selected from the corresponding lamina database. These properties were the localized MOEs and the calculated tensile strengths of lamina, which were then assigned to the virtually segmented lamina elements of GLT. Each lamina element in a same grade was assumed to be linked in the longitudinal direction. The next input properties taken sequentially from the database were given to the next link (lamina elements). This assigning process would be repeated to the last lamina element.

The positions of the finger joints were determined by KAR criterion. If a detected knot gave a bigger value than the maximum allowable KAR, the calculated tensile strength which was based on the KAR and MOE measurements was replaced with the tested tensile strength of finger joint. Laminae that are too short would not be finger-jointed in a real manufacturing process. Thus, a minimum spacing between finger joints was set to be $800 \mathrm{~mm}$ in the model.

\section{Predicting MOR of a virtual GLT}

The bending strength of a virtual GLT was predicted using composite beam theory applied for each segment. According to the composite beam theory, the stress in the $i$ th lamina is given under moment, $M$, by the following equation:

$\sigma_{t, i}=\left(\frac{E_{i} M y_{i, t}}{\left(\frac{w t^{3}}{12}\right) \sum_{i} E_{i}+(w t) \sum_{i} E_{i} y_{i, c}^{2}}\right)$,

where $\sigma_{t, i}$ is the tensile stress of the $i$ th lamina (MPa), $E_{\mathrm{i}}$ is the MOE of the $i$ th lamina (MPa), $M$ is the bending moment of the virtual GLT ( $\mathrm{N} \mathrm{mm}), y_{i, t}$ is the distance between a neutral axis of virtual GLT and the stress point of ith lamina $(\mathrm{mm}), w$ is the width of the lamina (mm), $t$ is the thickness of the lamina (mm), $E_{i}$ is the MOE of the $i$ th lamina (MPa),

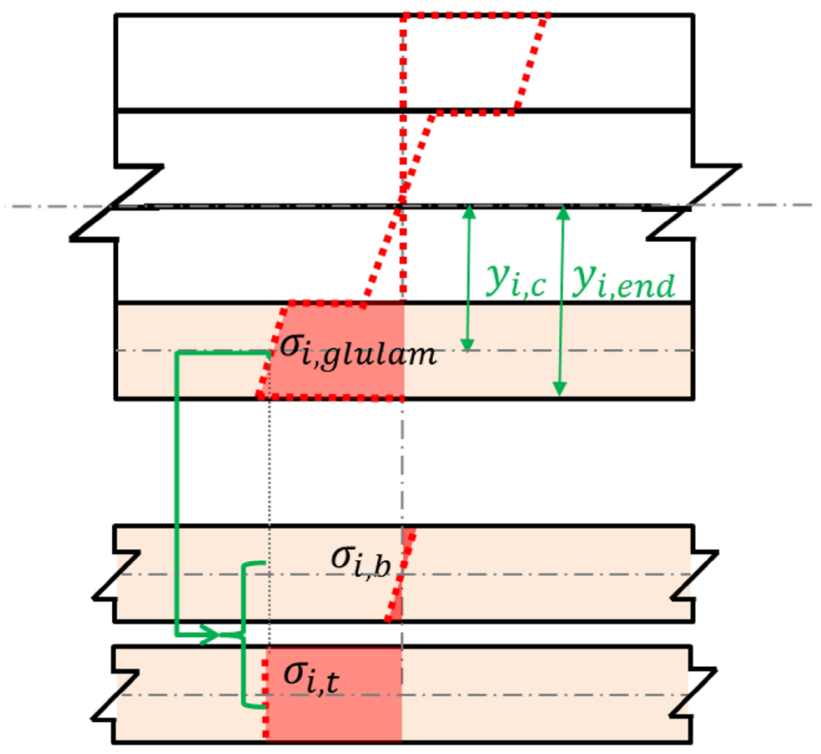

Fig. 1 Linear stress in GLT beam was considered combined stress by bending $\left(\sigma_{i, b}\right)$ and tension $\left(\sigma_{i, t}\right)$ in lamina

and $y_{i, c}$ is the distance between a neutral axis of the virtual GLT and a centroid of the $i$ th lamina (mm).

In Eq. (1), a distance between a neutral axis of virtual GLT and the calculation point of tensile stress of $i$ th lamina, $y_{i, t}$, was determined by Eq. (2). The tensile strength of the lamina was determined in a uniaxial tensile test. The tensile stress of a GLT in bending was not uniform through the depth of beam. The stress distribution in a GLT had a trapezoidal shape, as shown in Fig. 1. Thus, the distance between a neutral axis of virtual GLT and a calculation point of tensile stress profile in the lamina should be considered. Bender et al. [7] used the mid-depth stress criterion. Serrano et al. [8] showed that the mid-depth stress criterion is suitable only for large beam depths. Meanwhile, Mihashi et al. [9] compared three different criteria and showed that a combined failure criterion, modulus of rupture (MOR), and tensile strength gave the best agreement with the corresponding experimental results. In this study, $y_{i, t}$ was determined by Eq. (2) which was derived from the combined stress of bending and tension. Equation (2) and $k$ were considered to reflect the actual stress point in GLT. The references for $k$ show the ratio of bending and tensile strength by actual full-scale tests.

$y_{i, t}=\frac{\sqrt{\left(k y_{i, c}\right)^{2}+y_{i, c}{ }^{2}+y_{i, \text { end }}^{2}-2 y_{i, c} y_{i, \text { end }}}}{k}$,

where $y_{i, t}$ is the distance between a neutral axis of virtual GLT and a calculation point of tensile stress of ith lamina $(\mathrm{mm}), y_{i, c}$ is the distance between a neutral axis of virtual 
GLT and a centroid of $i$ th lamina (mm), $y_{i, \text { end }}$ is the distance between a neutral axis of virtual GLT and an outermost of ith lamina $(\mathrm{mm}), k$ is the ratio of bending and tensile strength of lamina, and herein, 1.45 was used as references [10-13].

The neutral axis of each segment was calculated using the localized MOE of each lamina element. The moment capacity of each segment was computed using the localized tensile strength of each lamina element [Eq. 3]. This procedure was repeated along the maximum moment zone which was a middle 1/3 span of a GLT beam under third-point loading. The minimum moment obtained from all calculations along the zone defined the ultimate moment-carrying capacity of the virtual GLT beam:

$M_{\mathrm{ult}, \mathrm{s}}=\min \left(\frac{f_{t, i}\left(\left(\frac{w t^{3}}{12}\right) \sum_{i} E_{i}+(w t) \sum_{i} E_{i} y_{i, c}^{2}\right)}{E_{i} y_{i, t}}\right)$,

where $M_{\mathrm{ult}, \mathrm{s}}$ is the ultimate moment carrying capacity of virtual GLT at a segment $(\mathrm{N} \mathrm{mm}), f_{t, i}$ is the tensile strength of the ith lamina (MPa), $w$ is the width of the lamina (mm), $t$ is the thickness of the lamina (mm), $E_{i}$ is the MOE of the $i$ th lamina (MPa), $y_{i, c}$ is the distance between a neutral axis of the virtual GLT and a centroid of ith lamina (mm), and $y_{i, t}$ is the distance between a neutral axis of virtual GLT and a calculation point of tensile stress of $i$ th lamina $(\mathrm{mm})$.

Once the ultimate moment-carrying capacity of the GLT beam was determined, the apparent MOR of the beam was calculated using the following equation:

$\mathrm{MOR}=\frac{M_{\mathrm{ult}}}{S}$,

where $M_{\text {ult }}$ is the ultimate moment carrying capacity of entire virtual GLT ( $\mathrm{N} \mathrm{mm}$ ), and $S$ is the the section modulus of the virtual GLT $\left(\mathrm{mm}^{3}\right)$.

\section{Experimental procedures}

\section{Input properties database}

\section{Localized MOE and KAR}

Samples of Japanese Larch (Larix kaempferi Carr., ovendry density: $540 \mathrm{~kg} / \mathrm{m}^{3}$ ) grown in Gangwon Province, Korea were used as specimens. After kiln drying, the surfaces were planed, so that the final cross sections were $38 \mathrm{~mm} \times 89 \mathrm{~mm}$ or $38 \mathrm{~mm} \times 140 \mathrm{~mm}$ with a length of $3600 \mathrm{~mm}$. The moisture content of the specimens was approximately $10-13 \%$. The moisture content was measured using microwave moisture content device (MB-3100, Japan). The specimens of lamina involved were listed in Table 1.
Table 1 Specimens for input property database

\begin{tabular}{llll}
\hline Dimensions (mm) & Grade $^{\mathrm{a}}$ & $\begin{array}{l}\text { Number of } \\
\text { specimens }\end{array}$ \\
\hline $38 \times 89 \times 3600$ & E8 (8-9 GPa of MOE) & 266 & 815 \\
& E10 (10-11 GPa of MOE) & 378 & \\
& E12 (12-13 GPa of MOE) & 171 & \\
$38 \times 140 \times 3600$ & E8 (8-9 GPa of MOE) & 246 & 830 \\
& E10 (10-11 GPa of MOE) & 352 & \\
& E12 (12-13 GPa of MOE) & 232 & \\
\hline
\end{tabular}

MOE modulus of elasticity

${ }^{a}$ E-rated grade which was determined by average of localized MOEs in a lamina

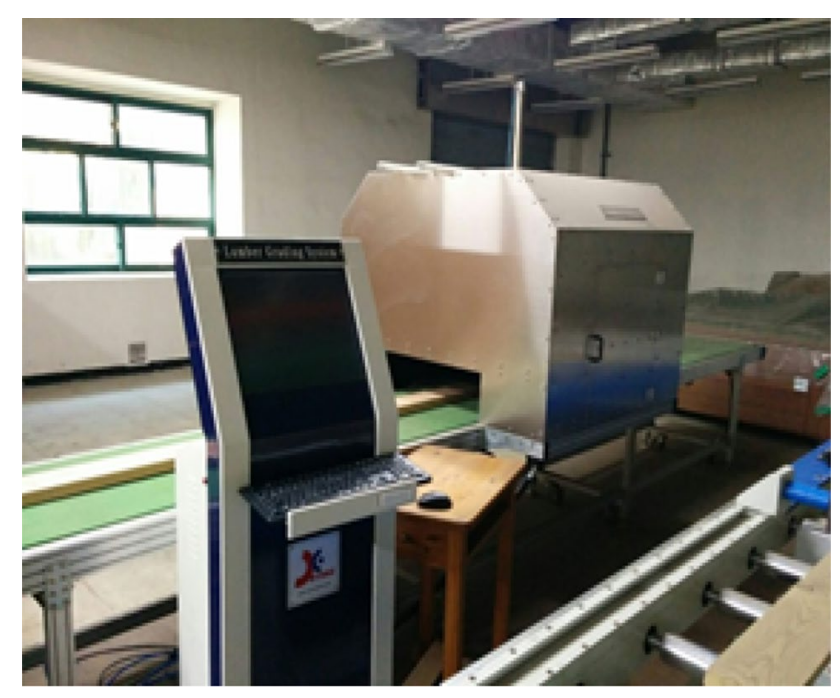

Fig. 2 Image-processing system (Kim et al. [14, 15])

The grades of lamina specimens were measured by machine stress-rated grader (MGFE-251, Japan). The localized MOE was recorded for reflecting the lengthwise variation in each lamina. The KAR was defined as the ratio of knot area to the cross-sectional area. Knot images on four surfaces of a lamina were captured using an image-processing system developed by Kim et al. [14, 15] (see Fig. 2). Knots were classified according to the knot area type in ASTM D3737 [16]. The knot area type is triangle or equilibrium quadrilateral shapes. The knot area was calculated using the knot length at perpendicular to longitudinal direction. The calculated knot area divided by cross-sectional area of lamina. The knot length was detected from the knot image and the KAR was automatically calculated using the Matlab software (2014a, The Mathworks, Natick, MA, USA). In this study, the knot position, central or edge, was not considered. 
Table 2 Specimens for tensile strength test

\begin{tabular}{llll}
\hline Dimensions $(\mathrm{mm})$ & Grade $^{\mathrm{a}}$ & \multicolumn{2}{c}{$\begin{array}{l}\text { Number of } \\
\text { specimens }\end{array}$} \\
\hline $38 \times 89 \times 1800$ & E8 (8-9 GPa of MOE) & 34 & 99 \\
& E10 (10-11 GPa of MOE) & 35 & \\
& E12 (12-13 GPa of MOE) & 30 & \\
$38 \times 140 \times 1800$ & E8 (8-9 GPa of MOE) & 12 & 220 \\
& E10 (10-11 GPa of MOE) & 107 & \\
& E12 (12-13 GPa of MOE) & 101 & \\
& & &
\end{tabular}

MOE modulus of elasticity

${ }^{a}$ E-rated grades are the same as for Table 1

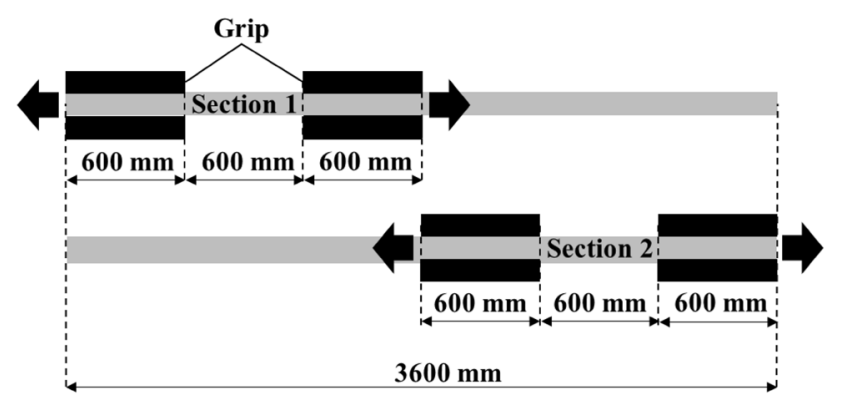

Fig. 3 Tensile test arrangement for in-grade tested lamina

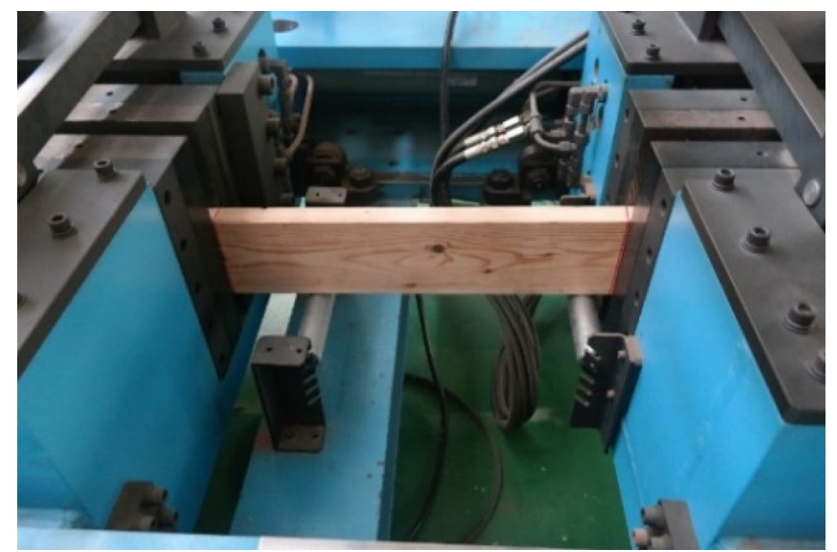

Fig. 4 Tensile strength test setup (Kyoungsung Testing Machine Co. LTD., Korea)

\section{Material model for generating tensile strength of a lamina}

To develop a material model for generating tensile strength from KAR and MOE data, tensile strength tests were carried out with the laminae, as shown in Table 2, which were randomly sampled from the specimens of Table 1 . The full length of the specimens was $3600 \mathrm{~mm}$. As shown in Fig. 3, the length of the grip for holding the specimen was $600 \mathrm{~mm}$, and the span of tension test between the grips was also $600 \mathrm{~mm}$. Thus, the tensile strengths of two parts in a lamina could be tested.

Figure 4 shows the tensile testing machine. The loading speed was $5 \mathrm{~mm} / \mathrm{min}$, which leaded the tests to be finished within $5 \mathrm{~min}$. The tensile strength was calculated using Eq. (5). Regarding the full-scale tensile strength test of lumber, Foschi and Barrett [1] revealed the importance of test configurations due to the lateral movement of edge-cracked specimen. Herein, to control the lateral movement, supports were installed on the bottom of specimens. Thus, the lateral movement of specimens would occur in the upper direction:

$f_{t, \text { lamina }}=\frac{P_{\max }}{w \times t}$,

where $f_{t, \text { lamina }}$ is the tensile strength of the lamina (MPa), $P_{\max }$ is the applied maximum load $(\mathrm{N}), w$ is the width of the lamina (mm), and $t$ is the thickness of the lamina (mm).

Two parameters, KAR and MOE, as estimator a linear multiple regression model (Eq. 6) were used for predicting tensile strengths of lamina. The regression coefficients were derived by the partial least squares (PLS) method using the Minitab software (Minitab Inc., PA, USA):

$Y=\beta_{0}+\beta_{1} X_{1}+\beta_{2} X_{2}+\varepsilon$,

where $Y$ is the predicted tensile strength (MPa), $\beta_{i}$ is the regression coefficients, $X_{1}$ is the independent variable (herein, knot area ratio), $X_{2}$ is the independent variable (herein, modulus of elasticity) (GPa), and $\varepsilon$ is the error term.

The localized KARs were matched with the localized MOE at the same position on the lamina; then, the tensile strength of $1 \mathrm{~mm}$-long lamina element was calculated using these localized KAR and MOE values. The 345 points of localized MOE were recorded by MSR machine and the measured length was $3000 \mathrm{~mm}$. Thus, the length of localized MOE was approximately $8.7 \mathrm{~mm}$ and the same MOE was applied within the corresponding section. Meanwhile, the detected image for measuring a KAR was continuous along the longitudinal direction of lamina. Knot lengths at perpendicular to the longitudinal direction on four surfaces of lamina were used for calculating KAR. The knot lengths can be detected at $1 \mathrm{~mm}$ intervals along the longitudinal direction. Thus, the localized tensile strength can be predicted for every $1 \mathrm{~mm}$-long element.

The both parameters (KAR and MOE) are size-independent, but the tensile strength varies depending on the species, grade, and size [17, 18]. Thus, the regression coefficients of Eq. (6) should be different depending on the species, grade, and size.

\section{Tensile strength of finger joints}

The ends of the laminae were connected with a finger joint. The tensile strength of the finger-jointed lamina was different 
Table 3 Specimens for tensile strength of finger-jointed lamina

\begin{tabular}{lllr}
\hline Dimensions (mm) & Grade $^{\mathrm{a}}$ & \multicolumn{2}{l}{$\begin{array}{l}\text { Number of } \\
\text { specimens }\end{array}$} \\
\hline $38 \times 89 \times 1800$ & E8 (8-9 GPa of MOE) & 30 & 120 \\
& E10 (10-11 GPa of MOE) & 30 & \\
$38 \times 140 \times 1800$ & E12 (12-13 GPa of MOE) & 30 & \\
& E8 (8-9 GPa of MOE) & 30 & 120 \\
& E10 (10-11 GPa of MOE) & 30 & \\
& E12 (12-13 GPa of MOE) & 30 & \\
\hline
\end{tabular}

MOE modulus of elasticity

${ }^{a}$ E-rated grades are the same as for Table 1

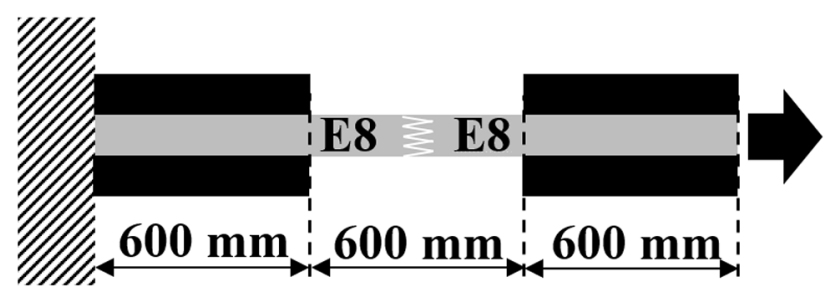

Fig. 5 Example of tensile test for finger-jointed lamina

from that of a lamina without finger joints. To derive the tensile strength distribution of finger-jointed lamina, 30 pieces of finger-jointed laminae were manufactured for each grade in Table 3. The same grade laminae were connected using finger joints which were positioned in the middle of the test span (see Fig. 5). The laminae for making finger joints were selected randomly in a same lamina stock, and cut to $900 \mathrm{~mm}$ from $3600 \mathrm{~mm}$ lamina. The $900 \mathrm{~mm}$ laminae were used for making finger-jointed lamina. Thus, some fingerjointed laminae, $1800 \mathrm{~mm}$ length, were fabricated from 1 original lamina, $3600 \mathrm{~mm}$ length. Some finger-jointed laminae were fabricated from two different original laminae in a same grade. The experimental procedure for measuring tensile strength for the finger-jointed lamina was the same with that for the non-finger-jointed lamina.

\section{Prediction of bending strength distribution}

A virtual GLT beam was modelled to simulate the experimental GLT beam. The calculation of the bending strength of the virtual GLT beam was carried out using the Matlab software (2014a, The Mathworks, Natick, MA, USA). One thousand virtual GLT beams were simulated. The localized MOE and KAR and the tensile strengths of the finger joints were incorporated into the model as input properties.

At first, the localized MOEs and KARs within each layer in virtual GLT were randomly picked up from the actual lamina database as long as the virtual GLT length. Second, localized tensile strengths were generated from the adjusted localized MOE and KAR using Eq. (6). Third, in manufacturing process of GLT, the ends of same lamina grades are connected with finger joint. Thus, finger-jointed strengths were applied according to lamina size and MOE lamina grade. The finger-jointed strengths were generated by the Weibull inverse cumulative distribution function. Weibull parameters for finger-jointed strengths were derived from full-scale tensile strength tests.

In manufacturing a finger-jointed lamina, knots that had a bigger KAR than approximately 0.4 were removed. The minimum length between finger joints was set as $800 \mathrm{~mm}$, because shorter lamina length would not be used in the manufacturing practice. Thus, virtual GLT beams with finger joints were generated having the following characteristics.

1) Tensile strength of lamina element having a bigger knot than 0.4 KAR was replaced with tensile strength of finger joint in the same grade. The KARs of extracted knot section from lamina for controlling the visual quality level were measured. The finger-joint worker instinctively cut a knot section which have larger knot than third of lamina width.

2) The minimum distance between finger joints was set as $800 \mathrm{~mm}$. If the lamina length was shorter than $800 \mathrm{~mm}$, the lamina was removed.

3) At least one finger joint was forcibly placed in the middle of the virtual GLT beam to simulate the actual GLT specimens.

\section{Experimental tests for model validation}

Four sets of GLT beams were manufactured for validating the GLT beam model. Set 1 was prepared for the GLT without finger joints. Set 2 was for the GLT with finger joints. Set 3 and Set 4 were intended to investigate whether the model capability maintained with the changes in the size/volume or the combinations of lamina grades of GLT, respectively. All laminae for manufacturing GLT were selected in random from the designated grade specimens for establishing input properties. However, after the planning process, the dimension of surfaced GLT specimen should be reduced from the

Table 4 Specimens for validating simulated GLT-bending strength distributions

\begin{tabular}{llrccl}
\hline & Finger joint & \multicolumn{2}{c}{ Final dimension $(\mathrm{mm})$} & \multirow{2}{*}{$\begin{array}{l}\text { Number of } \\
\text { specimens }\end{array}$} \\
\cline { 3 - 5 } & & Width & Depth & Length & \\
\hline Set 1 & No finger joint & 80 & 130 & 2400 & 30 \\
Set 2 & With finger joint & 80 & 130 & 2400 & 30 \\
Set 3 & With finger joint & 130 & 250 & 4800 & 30 \\
Set 4 & With finger joint & 80 & 250 & 4800 & 30 \\
\hline
\end{tabular}

Glued-laminated timber 
Fig. 6 Moment diagram with test setup for measuring bending strength

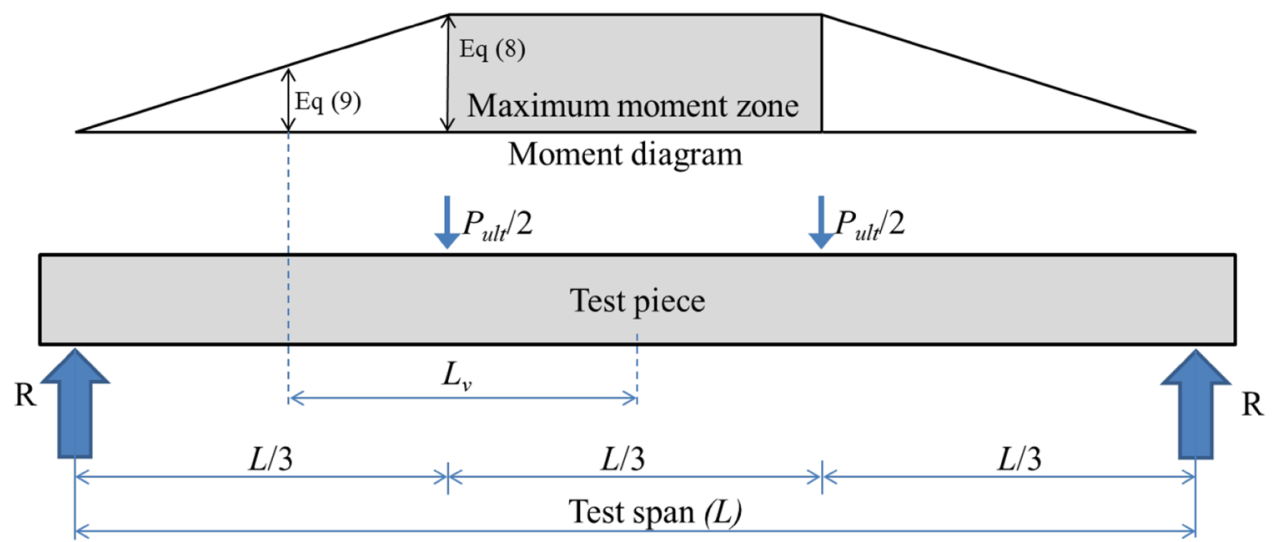

sum of the original lamina depth. The final dimensions of GLT specimens for model validation are shown in Table 4.

A third-point loading test was carried out using a universal testing machine (Zwick GmbH \& Co., Ltd., Ulm, Germany) to evaluate the bending strength of the GLT (see Fig. 6) [19]. The span to depth ratio was 1:18, and the load speed was $5 \mathrm{~mm} / \mathrm{min}$. Specimen failure occurred within $5 \mathrm{~min}$. The MOR was calculated from the maximum bending moment, as given in the following equation [19]:

$\mathrm{MOR}=\frac{M_{\max } y}{I}=\frac{6 M_{\max }}{w_{\text {test }} t_{\text {test }}^{2}}$,

where $M_{\max }$ is the maximum bending moment ( $\left.\mathrm{N} \mathrm{mm}\right), y$ is the distance from the neutral axis of the GLT beam ( $\mathrm{mm}$ ), $I$ is the moment of inertia of the cross section $\left(\mathrm{mm}^{4}\right), w_{\text {test }}$ is the width of the test piece $(\mathrm{mm})$, and $t_{\text {test }}$ is the thickness of the test piece $(\mathrm{mm})$.

The maximum bending moment was calculated within maximum moment zone (MMZ) using the following equation:

$M_{\max \text { in MMZ }}=\frac{P_{\max } L}{6}$,

where $M_{\max \text { in MMZ }}$ is the maximum bending moment in maximum moment zone $(\mathrm{N} \mathrm{mm}), P_{\max }$ is the applied maximum load $(\mathrm{N})$, and $L$ is the test span $(\mathrm{mm})$.

\section{Results and discussion}

\section{Input properties database}

\section{MOE and tensile strength profiles of lamina}

As shown in Fig. 7, the MOE profiles of laminae were obtained using MSR machine. The image-processing system recorded the KARs in laminae. In Fig. 7, the gray zone shows the tensile strength test zone, $600 \mathrm{~mm}$, as shown in
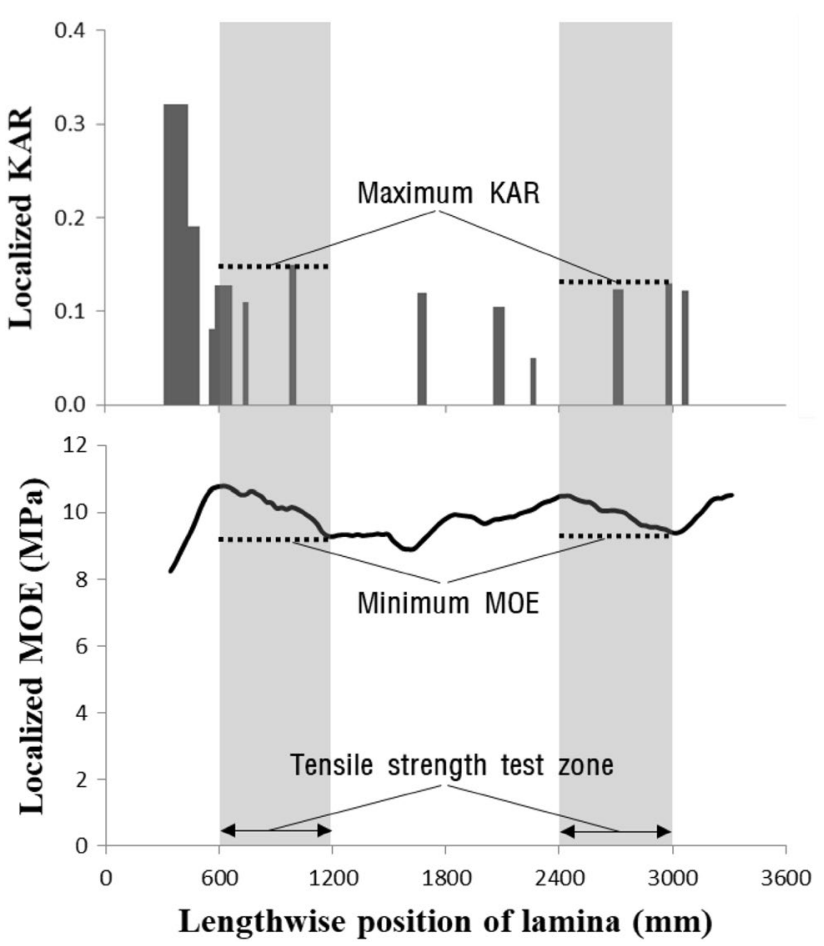

Fig. 7 Example of localized KAR and MOE data used for deriving a material model. Note. the gray zones (600-1200 and $2400-3000 \mathrm{~mm}$ on the $x$-axis) show the tensile strength test zone, as shown in Fig. 3. The black dotted lines parallel to $x$-axis show the maximum localized $\mathrm{KAR}$ and the minimum localized MOE in the test zone

Fig. 3. The horizontal black bar shows the maximum localized KAR and the minimum localized MOE in the test span. To generate the tensile strength of lamina elements, the maximum KAR and the minimum MOE in the tensile test span were used. In the case of a specimen that has small knots, a failure in tension often occurred around the grip. The tested specimens with this grip failure were excluded, because the objective of the test was to obtain the relationship between KAR and tension strength (see Fig. 8). The 
number of specimens in Table 2 was counted only for the failure around knots. The lateral movement of specimens was not observed.

When using multiple regression, the coefficient estimates can change erratically in response to small changes in the model or the data. Thus, multicollinearity, a phenomenon in which two or more predictive variables are highly correlated, should be checked. PLS regression was carried out to check the main components and drive the fit to a more reliable regression. This would get rid of the multicollinearity problem [20]. The standardized coefficients and the relative importance of each predictor in the model showed that KAR was a main component, as shown in Table 5. When adding MOE to the fit, the multiple determination, $R^{2}$ increased. This means that the consideration of both MOE and KAR was optimal for predicting tensile strength. The $R^{2}$ value indicates how much the change of a dependent variable is caused by the change of the independent ones. These values were 61-63\% (see Fig. 9). Finally, the database of MOEs and tensile strength of lamina was constructed at each grade.

Fig. 8 Failure modes in tensile strength test

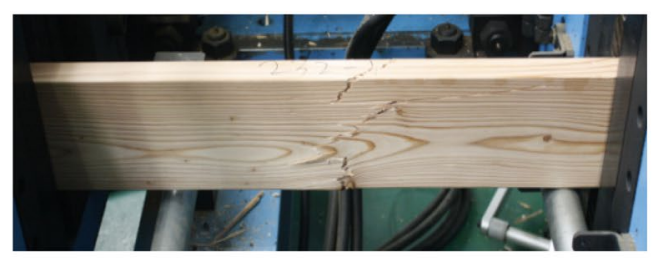

(a) Failure caused by knot

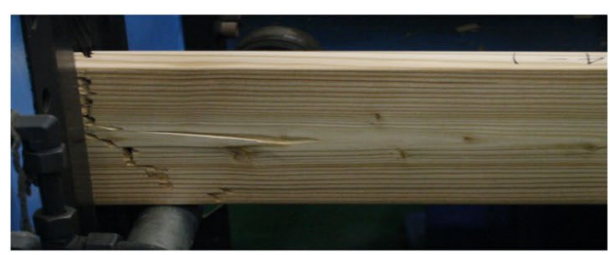

(b) Failure caused by grip
Table 5 Partial least squares multiple regression results for KAR, MOE, and tensile strength

\begin{tabular}{llcccc}
\hline Lamina size $(\mathrm{mm})$ & Predictor & Coefficient & $\begin{array}{l}\text { Standardized } \\
\text { coefficient }\end{array}$ & $p$ value $^{\mathrm{a}}$ & $R^{2 \mathrm{~b}}$ \\
\hline $38 \times 89$ & Constant & 41.23 & & $1.78 \times 10^{-10}$ & \\
& KAR & -69.71 & -0.62 & $6.27 \times 10^{-16}$ & 0.55 \\
& MOE (GPa) & 1.63 & 0.30 & $7.85 \times 10^{-4}$ & 0.61 \\
$38 \times 140$ & Constant & 39.40 & & $9.58 \times 10^{-9}$ & \\
& KAR & -56.38 & -0.67 & $2.73 \times 10^{-19}$ & 0.61 \\
& MOE (GPa) & 1.26 & 0.19 & $3.69 \times 10^{-3}$ & 0.63 \\
\hline
\end{tabular}

Knot area ratio

Modulus of elasticity

${ }^{a}$ The statistical uncertainties between dependent variable and independent ones

${ }^{\mathrm{b}}$ The coefficient of determination between dependent variable and independent ones. This value indicates how much the change of a dependent variable is caused by the change of the independent ones

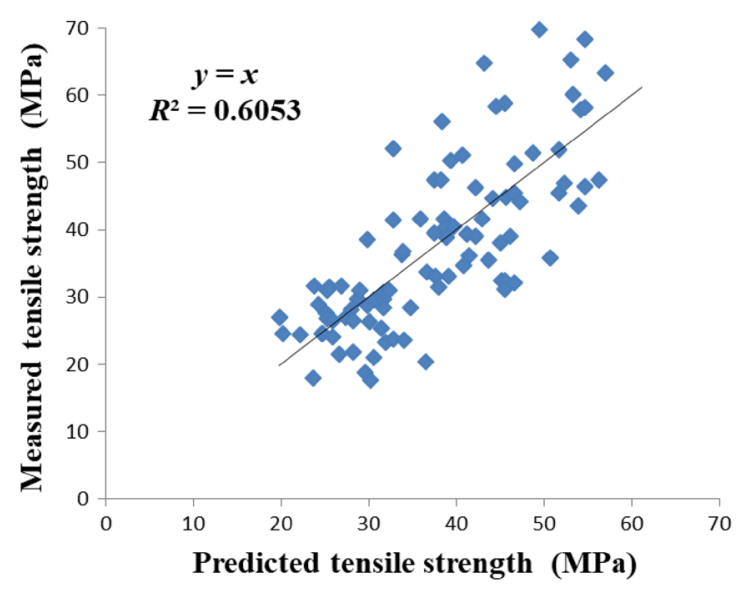

(a) $38 \mathrm{~mm} \times 89 \mathrm{~mm}$

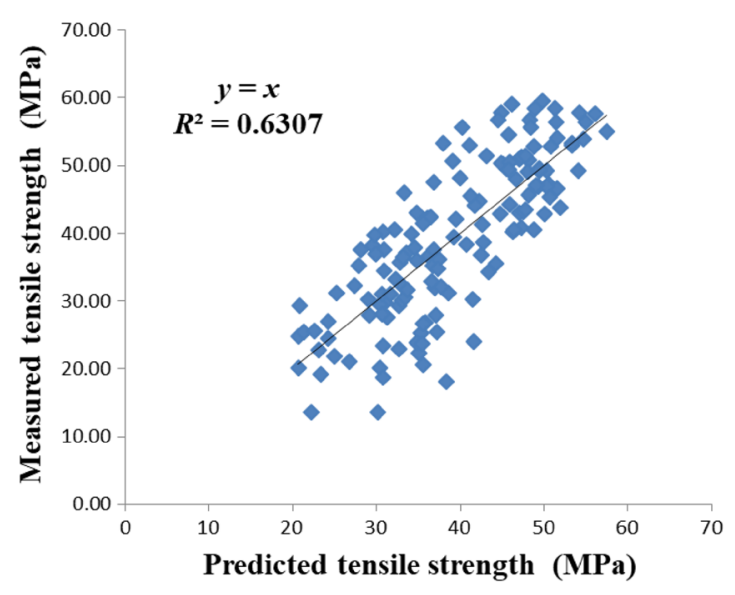

(b) $38 \mathrm{~mm} \times 140 \mathrm{~mm}$

Fig. 9 Comparison between measured and predicted tensile strength using multiple regression 


\section{Tensile strength distribution of finger-jointed lamina}

The tensile strength distributions of finger-jointed lamina in grades are shown in Fig. 10. The distribution of higher lamina grade fell to the right of lower grade lamina. The percentage of grip failure was decreased with increasing lamina grade and size. The finger-jointed lamina is known that a reduction in strength is $15-25 \%$ compared to the small clear specimens [21]. Thus, the failure ratio at finger joint was high in the high quality grade which has low defects.

The developed model focuses on the lower tail fitting for deriving design values of GLT. Some standards use $15 \%$ lower tail distribution for deriving $5 \%$ point estimate

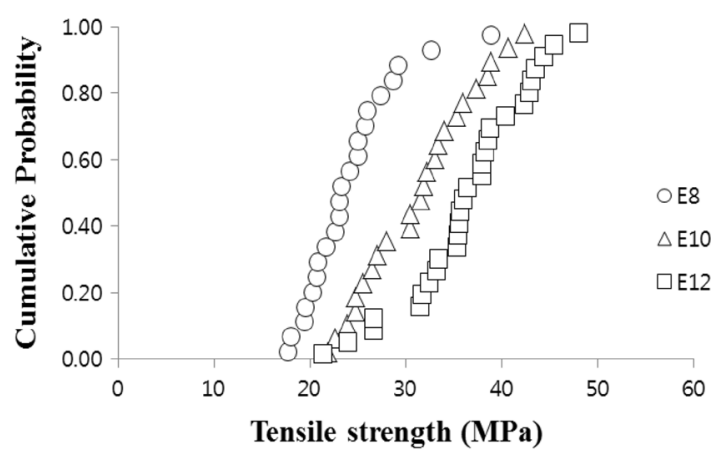

(a) $38 \mathrm{~mm} \times 89 \mathrm{~mm}$
[22-24]. To better predict the lower distribution of finger joint, the distribution was fitted to the $15 \%$ lower tail test data. Moreover, the different distributions were applied according to lamina size and grade, as shown in Table 6.

\section{Validation of glued-laminated timber model}

\section{Validation of GLT without finger joints (Set 1)}

One thousand virtual GLT beams were generated from the input database of $38 \mathrm{~mm} \times 89 \mathrm{~mm}$ lamina stock. The virtual GLT beams in Set 1 did not have finger joints and were made using two lamina grades of E12 and E8 (see Fig. 11a). The

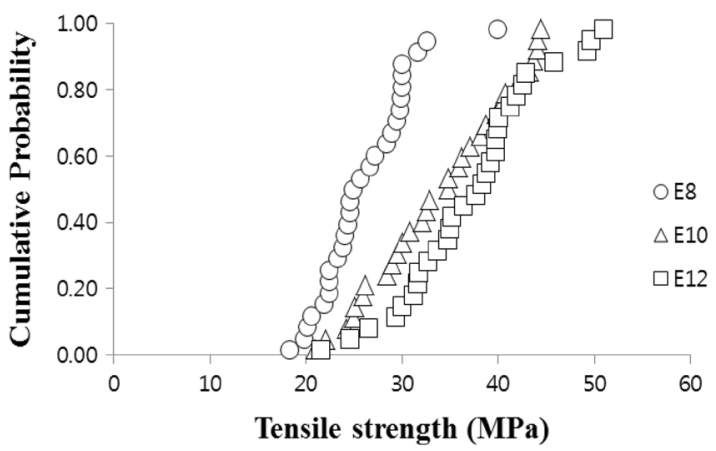

(b) $38 \mathrm{~mm} \times 140 \mathrm{~mm}$

Fig. 10 Tensile strength distribution of finger-jointed lamina as a function of specimen size

Table 6 Distribution parameters of tensile strengths for finger joint

\begin{tabular}{|c|c|c|c|c|c|c|c|c|}
\hline \multirow[t]{2}{*}{ Lamina size (mm) } & \multirow[t]{2}{*}{ Grade $^{\mathrm{a}}$} & \multicolumn{3}{|c|}{ Percentage of failure (\%) } & \multirow[t]{2}{*}{$5 \% \mathrm{NPE}^{\mathrm{b}}$} & \multicolumn{3}{|c|}{ 2P-Weibull ${ }^{\mathrm{c}}$} \\
\hline & & Finger joint & Knot & Grip & & $5 \% \mathrm{WPE}^{\mathrm{d}}$ & Shape & Scale \\
\hline \multirow[t]{3}{*}{$38 \times 89$} & E8 & 66.7 & 6.7 & 26.7 & 17.81 & 16.78 & 6.97 & 25.69 \\
\hline & E10 & 73.3 & 10.0 & 16.7 & 22.39 & 21.49 & 6.73 & 33.40 \\
\hline & E12 & 100 & 0 & 0 & 23.56 & 24.58 & 6.49 & 38.85 \\
\hline \multirow[t]{3}{*}{$38 \times 140$} & E8 & 73.3 & 16.7 & 10.0 & 19.72 & 18.64 & 7.50 & 27.70 \\
\hline & E10 & 80.6 & 12.9 & 6.5 & 22.20 & 21.96 & 5.81 & 36.61 \\
\hline & E12 & 100 & 0 & 0 & 24.54 & 24.70 & 6.24 & 39.76 \\
\hline
\end{tabular}

${ }^{a}$ E-rated grades are the same as for Table 1

${ }^{b}$ Non-parametric fifth percentile point estimate

${ }^{\mathrm{c}}$ Two parameter (2P) Weibull distribution

${ }^{\mathrm{d}}$ Parametric fifth percentile point estimate using Weibull distribution fit

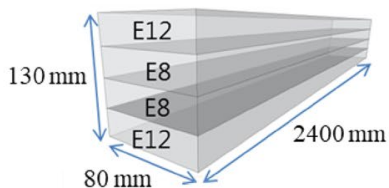

(a) Set 1 and 2

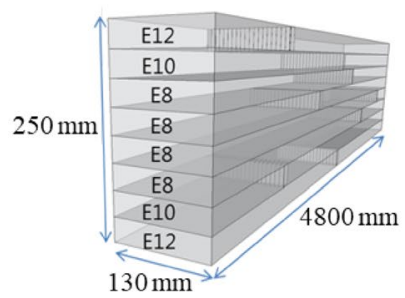

(b) Set 3

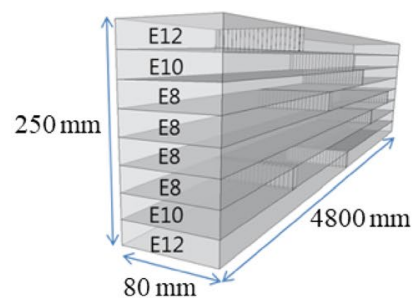

(c) Set 4 
Fig. 12 Figure modes of GLT

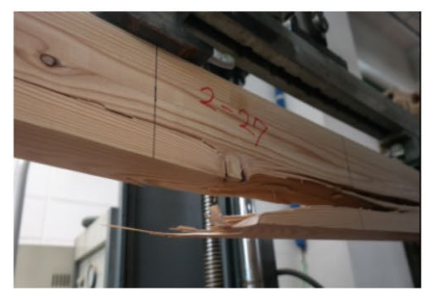

(a) Tension failure around knot in tension zone

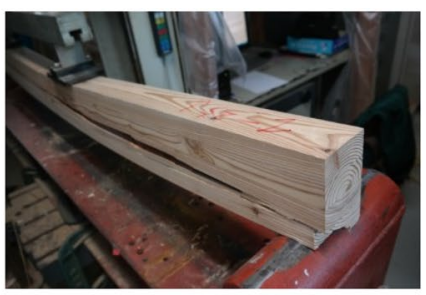

(b) Shear failure

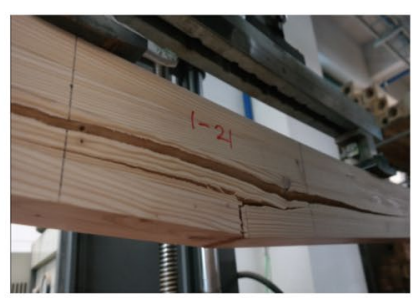

(c) Finger-joint failure three lamina grades were selected to set a 10S-30B GLT grade which is the most commonly used GLT grade in Korea. The layup of lamina grades corresponds to the 10S30B GLT grade in KS F 3021 [25]. Thirty actual GLT beams (Set 1) were manufactured. The bending tests were carried out for validating the simulated bending strength distribution of GLT. Most GLT specimens (27 of them) failed around a knot in the tension zone (see Fig. 12a). Meanwhile, shear failure appeared in three GLT specimens (see Fig. 12b). The maximum moment-carrying capacities of the shear-failed specimens were much higher than the knot-failed specimens. The shear failure might be induced by a high tensile strength of the outermost lamina. Although the model could not capture shear failure, it would be acceptable, since a high tensile strength of the outermost lamina also gave a high chance to produce the high moment-carrying capacity in the model. It means that the failure types in simulation would not be an influential factor in the prediction of distribution.

The Kolmogorov-Smirnov two-sample test (K-S test) was performed to determine whether the simulated and actual cumulative distribution functions were significantly different, as shown in Table 7. There was not sufficient evidence to conclude that the two distributions were different at significance level of 0.01 . The difference of the non-parametric $5 \%$ point estimate was approximately $1 \%$. As shown in Fig. 13a, overall, the simulated distribution of bending strength fitted the test data. Especially, good agreement was found in the lower tail of the distribution. However, the upper tail of the distribution was deviated from the test data. Although a clear explanation about the deviation could not be sought, it was thought that more test data might improve the results.

\section{Validation of GLT containing finger joints (Set 2)}

To ensure that the GLT model accurately accounts for the effect of finger joints in lamina, one thousand virtual GLT beams were generated from the input database of $38 \mathrm{~mm} \times 89 \mathrm{~mm}$ lamina stock. Thirty pieces of actual GLT beams that included finger joints in the lamina were manufactured. The layup of the lamina grades was the same as that in Set 1 (see Fig. 11a). Finger joints were placed in the middle of the outmost lamina in GLT specimens to induce failures in finger joints. Most of the failure in the actual GLT specimens (28 of them) occurred in the finger joints (see Fig. 12c). The failure around the knots in the tension zone was observed in only two test specimens, since severe knots in lamina were cut and replaced with finger joints or, rare within the short maximum moment zone of $0.8 \mathrm{~m}$.

$\mathrm{K}-\mathrm{S}$ test indicated that the estimated distribution was not significantly different from the measured distribution at a significance level of 0.01. Especially, the comparison of the bending strength distributions showed that the model estimation fitted well at lower tail (see Fig. 13b). The difference of the non-parametric 5\% point estimate was approximately 1.1\%. The differences between Set 1 (see Fig. 13a) and 2

Table 7 Simulation and actual test results for glued-laminated timber

\begin{tabular}{|c|c|c|c|c|c|c|c|c|}
\hline & \multicolumn{2}{|l|}{ Set 1} & \multicolumn{2}{|l|}{ Set 2} & \multicolumn{2}{|l|}{ Set 3} & \multicolumn{2}{|l|}{ Set 4} \\
\hline & Test data & Simulated data & Test data & Simulated data & Test data & Simulated data & Test data & Simulated data \\
\hline Number of specimens & 27 & 1000 & 30 & 1000 & 30 & 1000 & 30 & 1000 \\
\hline $\begin{array}{l}\text { Non-parametric } 5 \% \text { point estimate } \\
(\mathrm{MPa})\end{array}$ & 40.83 & 41.22 & 32.67 & 30.41 & 31.91 & 29.06 & 31.02 & 30.98 \\
\hline Coefficient of variation & 0.22 & 0.17 & 0.18 & 0.25 & 0.15 & 0.16 & 0.16 & 0.15 \\
\hline $\mathrm{K}-\mathrm{S}^{\mathrm{a}}$ test statistic & 0.265 & & 0.259 & & 0.137 & & 0.19 & \\
\hline $\begin{array}{l}\text { K-S critical value } \\
\text { (significance level: } 0.01 \text { ) }\end{array}$ & 0.317 & & 0.302 & & 0.302 & & 0.302 & \\
\hline
\end{tabular}

${ }^{\mathrm{a}}$ Kolmogorov-Smirnov 


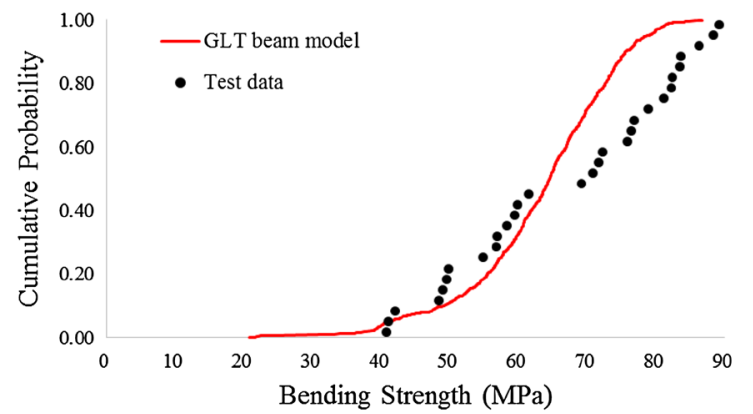

(a) Set 1

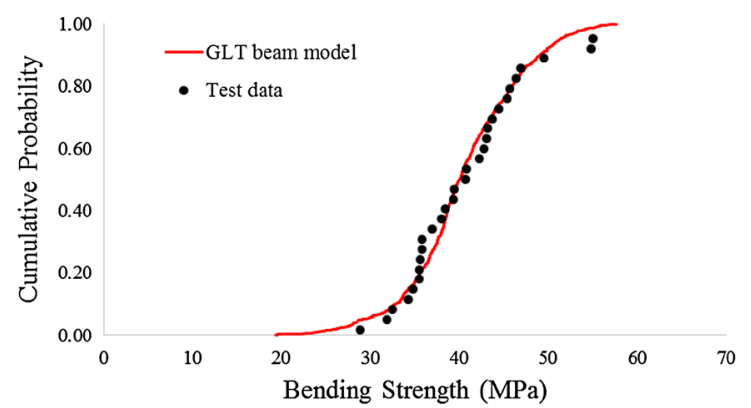

(c) Set 3

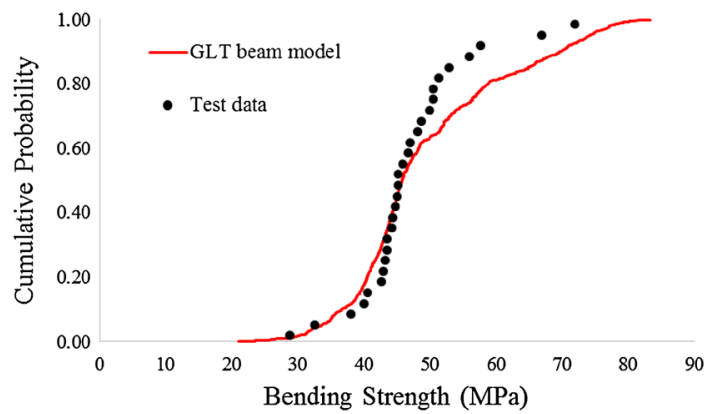

(b) Set 2

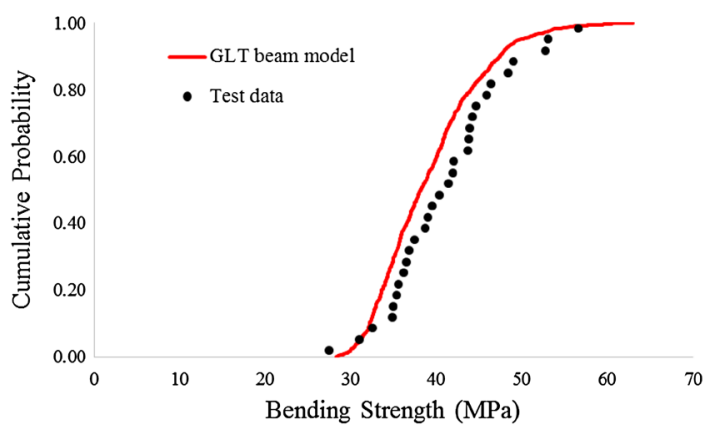

(d) Set 4

Fig. 13 Comparison of the simulated and tested bending strength distribution

(see Fig. 13b) were up to about $15 \mathrm{MPa}$ for the higher fractile values. It was caused by the strength of finger-jointed lamina. The strength range of finger-jointed lamina (see Fig. 10) was shorter than that of non-finger-jointed lamina (see Fig. 9).

\section{Validation of the different GLT sizes (Set 3)}

It is recognized that the mechanical properties of wood material are dependent on the size. The size/volume effects of solid wood have been explored by several researchers [26, 27]. The simulation of Set 3 was intended to investigate the model capability of simulating the size/volume-dependent bending strength of GLT.

One thousand virtual GLT beams were generated from the input database of $38 \mathrm{~mm} \times 140 \mathrm{~mm}$ lamina stock. The virtual GLT beams included finger joints and three lamina grades of E12, E10, and E8 (see Fig. 11b). The layup of lamina grades corresponds to the 10S-30B GLT grade in KS F 3021 [25] like Set 1 and 2. The layup of lamina grades is different depending on the GLT thickness to set the same GLT grade. Thirty actual GLT beams were manufactured for validating the simulated bending strength. The bending test results show that 20 of the specimens failed around finger joints and 10 GLT specimens failed around the knot. All of the failures occurred in the tensile zone, and the bending strength was governed by knots or finger joints.
The simulated distribution of bending strength matched the experimental results quite well (see Fig. 13c). The difference of the non-parametric 5\% point estimate was approximately $1.1 \%$.

\section{Validation for different combinations of lamina layers (Set} 4)

Set 4 (one grade layup) was prepared for simulating a GLT with a different grade combination of lamina from other Sets (symmetrical grade layups). The layup of lamina grades corresponds to 7S-27B GLT grade in KS F 3021 [25]. Thirty GLT specimens were manufactured for actual bending tests. One thousand virtual GLT beams were generated with the E8 lamina grade of $38 \mathrm{~mm} \times 89 \mathrm{~mm}$ lamina stock from the input database (see Fig. 11c). The bending test results of the actual GLT beams show that 12 specimens failed around finger joints and 18 specimens failed around the knot. All of the failures occurred in the tensile zone, and the bending strength of the GLT was governed by knots or finger joints.

The simulated distribution of bending strength was fit well with the test data (see Fig. 13d). The difference of the non-parametric $5 \%$ point estimate was approximately $1.1 \%$. The tested data were little bit concentrated on the right side of the simulated distribution.

The developed GLT model, in this study, predicts the bending strength of GLT using the tensile strength of 
weakest cross section. High strength for clear segment would be underestimated, but low strength for knot segment is not underestimated. Although the underestimated strength is assigned to clear segment, the assigned strength is higher than the strength for weak section. Thus, the underestimated strength for clear segment did not significantly govern the bending strength of GLT.

Meanwhile, the tensile strength of wood is size dependent. Moreover, due to the nature of wood, relationship between the parameters and tensile strength could be changed depending on the origin of tree, species, grade, and size. Thus, to show these concepts symbolically, in this study, individual parameters (KAR and MOE) were adopted for simulation. However, the both parameters are size-independent. It means that one combined model can be developed by including a size term. The combined model can be developed using statistical approach for various lamina sizes in further research.

\section{Conclusion}

In this study, a new GLT beam model was developed with consideration of the localized KARs and MOEs at the same position in a lamina. In addition, the effect of finger joints was incorporated into the model development. The main objective of this model was to estimate the bending strength distribution of actual GLT beams. For model validation, an experimental program on the bending tests of GLT was conducted. The program included four sets of GLT specimens which were classified depending on the inclusion of finger joint, the size of lamina, and the combination of lamina grades. The simulation results showed good agreement with the experimental data in the distributions and the fifth percentile bending strength. Therefore, it was thought that this developed model could be used in the development of GLT design value, size/volume effect factor and GLT strength grade provided that the required input properties of lamina were sufficiently ready.

\section{References}

1. Foschi RO, Barrett JD (1980) Glued-laminated beam strength: a model. J Struct Div 106(8):1735-1754

2. Hernandez R, Bender DA, Richburg BA, Kline KS (1992) Probabilistic modeling of glued-laminated timber beams. Wood Fiber Sci 23(3):294-306

3. Lee JJ, Park JS, Kim KM, Oh JK (2005) Prediction of bending properties for structural GLT using optimized distributions of knot characteristics and laminar MOE. J Wood Sci 51(6):640-647

4. Fink G, Frangi A, Kohler J (2014) Modelling the bending strength of glued-laminated timber-using machine-grading indicators. In: Proceeding of international network on timber engineering research, meeting 47, pp 371-374
5. Kandler G, Fussl J, Serrano E, Eberhardsteiner J (2015) Effective stiffness prediction of GLT beams based on stiffness distributions of individual lamellas. Wood Sci Technol 49(6):1001-1121

6. Serrano E (2003) Mechanical performance and modelling of GLT. In: Thelandersson S, Larsen HJ (eds) Timber engineering. Wiley, Chichester, pp 67-79

7. Bender DA, Woeste FE, Schaffer EL, Marx CM (1985) Reliability formulation for the strength and fire endurance of glued laminated beams. Research paper FPL460. Forest Products Laboratory, Madison

8. Serrano E, Gustafsson J, Larsen HJ (2001) Modeling of fingerjoint failure in glued laminated timber beams. J Struct Eng 127(8):914-921

9. Mihashi H, Itagaki N (1998) Probabilistic design of performance in glued laminated timber. In: PROBAMAT-21st Century: probabilities and materials, pp 333-345

10. Nakamura N, Fujita K (2011) Simulation method to generate the strength of GLT using correlated random variables. J Wood Sci 57(3):203-207

11. Forest Products Society (1997) Machine-graded lumber. Madison, WI: Forest Products Society. Wood Design Focus 8(2):1-24

12. West Coast Lumber Inspection Bureau (2004) Tension/Bending ratios of machine stress-rated lumber. Technical Report No. 2:8

13. Barrett JD, Jones ED, Lau W (1994) Canadian lumber properties. Canadian Wood Council, Ottawa, p 81

14. Kim BN, Lee HW, Kim KM (2009) The development of image processing system using area camera for feeding lumber. J Korean Wood Sci Technol 37(1):37-47

15. Kim BN, Kim KM, Shim KB, Lee HW, Shim SR (2009) The verification of image merging for lumber scanning system. J Korean Wood Sci Technol 37(6):556-565

16. ASTM D3737 (2008) Standard practice for establishing allowable properties for structural glued laminated timber (Glulam). American Society for Testing and Methods, West Conshohosken

17. Bender DA, Woeste FE (2012) Effect of variability on lumber design values. Frame Build News 24(4):34-39

18. Hoyle RJ, Galligan WL (1979) Characteristic lumber properties for truss research. In: Metal plate wood truss conference proceedings. Forest Products Society, Madison, WI, pp 79-28

19. Australian/New Zealand Standard 4063.1 (2010) Characteristic of structural timber part 1: test methods. Standards New Zealand, Wellington

20. Pirouz DM (2006) An overview of partial least square. Technical report, The Paul Merage School of Business, University of California, Irvine

21. Hoadley RB (2000) Understanding wood: a craftsman's guide to wood technology. The Taunton Press, Newtown

22. ISO 13910 (2005) Structural timber-characteristic values of strength-graded timber-sampling, full-size testing and evaluation. International Organization for Standardization, Geneva

23. Australian/New Zealand Standard 4063.2 (2010) Characteristic of structural timber part 2: determination of characteristic values. Standards New Zealand, Wellington

24. Barrett JD, Lau W (1994) Canadian lumber properties. Canadian Wood Council, Ottawa

25. KS F 3021 (2013) Structural glued laminated timber. Korean Standards Association, Seoul

26. Moody R, Falk R, Williamson T (1990) Strength of glulam beamssize effects. In: Proceedings of the 1990 international timber engineering conference, pp 176-182

27. Shedlauskas JP, Manbeck HB, Janowiak JJ, Hernandez R, Moody RC, Labosky JrP, Blankenhorn PR (1996) Efficient use of red oak for glued-laminated beams. Trans ASAE 39(1):203-209 